\title{
All reversible dynamics in maximally non-local theories are trivial
}

\author{
David Gross,,$* *$ Markus Müller, 2,3,† Roger Colbeck, ${ }^{4,5, \dagger}$ and Oscar C. O. Dahlsten ${ }^{4, \S}$ \\ ${ }^{1}$ Institute for Theoretical Physics, Leibniz University Hannover, 30167 Hannover, Germany \\ ${ }^{2}$ Institute of Mathematics, Technical University of Berlin, 10623 Berlin, Germany \\ ${ }^{3}$ Institute of Physics and Astronomy, University of Potsdam, 14476 Potsdam, Germany \\ ${ }^{4}$ Institute for Theoretical Physics, ETH Zurich, 8093 Zurich, Switzerland \\ ${ }^{5}$ Institute of Theoretical Computer Science, ETH Zurich, 8092 Zurich, Switzerland
}

(Dated: 1st March 2010)

\begin{abstract}
A remarkable feature of quantum theory is non-locality (i.e. the presence of correlations which violate Bell inequalities). However, quantum correlations are not maximally non-local, and it is natural to ask whether there are compelling reasons for rejecting theories in which stronger violations are possible. To shed light on this question, we consider post-quantum theories in which maximally non-local states (non-local boxes) occur. It has previously been conjectured that the set of dynamical transformations possible in such theories is severely limited. We settle the question affirmatively in the case of reversible dynamics, by completely characterizing all such transformations allowed in this setting. We find that the dynamical group is trivial, in the sense that it is generated solely by local operations and permutations of systems. In particular, no correlations can ever be created; non-local boxes cannot be prepared from product states (in other words, no analogues of entangling unitary operations exist), and classical computers can efficiently simulate all such processes.
\end{abstract}

Introduction.-Quantum mechanics exhibits the remarkable feature of non-local correlations, as highlighted in Bell's seminal paper [1]. Such correlations have (up to a few remaining loopholes) been extensively verified in experiments [2].

Aside from their theoretical importance, non-local correlations can be exploited for technological use: they are vital in entanglement-based quantum key distribution schemes [3], for example, where their presence can be used to guarantee security (see also [4] for a recent review).

While quantum mechanics violates Bell inequalities, it does not do so in the maximal possible way. There are conceivable devices, so-called non-local or Popescu-Rohrlich boxes, that permit even stronger correlations than quantum mechanics does, while respecting the no-signalling principle [5-77]. Such correlations are not observed in nature and the question arises as to whether other fundamental principles might be violated if they were to exist.

There has already been some progress towards answering this question. For example, the existence of non-local boxes would lead to some communication complexity problems becoming trivial [8, 9], the possibility of oblivious transfer [10] and the lack of so-called information causality [11]. It has also been realized that in a theory in which maximally Bell violating correlations emerge, the set of possible dynamical transformations would be severely restricted compared to those allowed in quantum theory [12]. While a complete classification of the dynamics has remained elusive, it has been shown, for example, that entanglement swapping is impossible [13, 14]. Furthermore, the question of the computational power of such a theory has been raised [12, 14].

We work in the framework of generalized probabilistic the-

\footnotetext{
*Electronic address: david.gross@ @itp.uni-hannover.de

${ }^{\dagger}$ Electronic address: mueller@math.tu-berlin.de

${ }^{\ddagger}$ Electronic address: colbeck@ phys.ethz.ch

§Electronic address: dahlsten@phys.ethz.ch
}

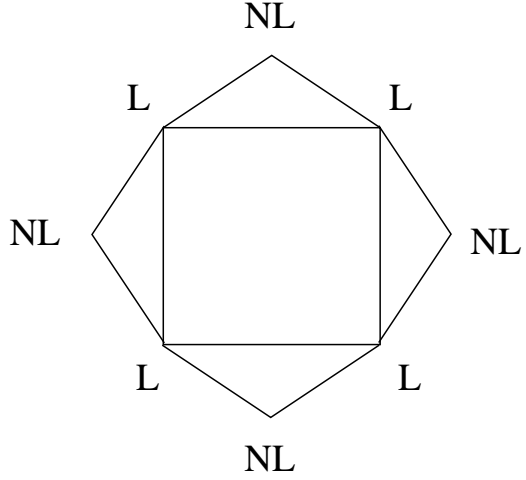

FIG. 1: Two-dimensional caricature of the (normalized) boxworld state space formed by stellating a square. Local vertices are denoted by $\mathrm{L}$ and non-local ones by NL. No symmetries of this object take L states to NL states or vice versa.

ories [12, 15-17], adopting the pragmatic operational view that the physical content of a theory is in the predicted statistics of measurement outcomes given preparations and transformations. The framework makes minimal assumptions and allows for mathematical rigour. We consider a system composed of $N$ subsystems. To each subsystem one of $M \geq 1$ measurements may be applied, yielding one of $K \geq 2$ outcomes (in the following, unless otherwise stated, we assume each subsystem has the same $M$ and $K$ ). The state space contains all non-signalling correlations, corresponding to socalled generalized non-signalling theory [12] or, more colloquially, boxworld.

Our main result (Theorem 1) is that (except in the case $M=1$ which corresponds to classical theory) the set of reversible transformations in boxworld is trivial: all such operations are a combination of local operations on a single system (which correspond to relabellings of measurements and their outcomes) and permutations of local systems (which correspond to relabellings of subsystems). This solves the afore- 
mentioned open problem concerning the computational power of boxworld in the case of reversible dynamics [12, 14].

Another interesting consequence is that, in boxworld, measurements and dynamics are necessarily distinct physical processes, in the sense that a measurement cannot be seen as a reversible dynamics on the system comprising the state and measurement device (cf. quantum theory, where the measurement process can be seen as a unitary evolution from the point of view of an external observer). We discuss this further in the final section.

We note that, in the case of a classical-boxworld hybrid system, Theorem 1 does not hold-we give an example of a CNOT operation on this system at the end of the paper. However, for all types of system, including those where the number of measurements and outcomes differs among the subsystems, reversible dynamics map pure product states to pure product states - that is, non-local states cannot be reversibly prepared from product states. This is our second main result (Theorem 2).

A geometric intuition behind this result is as follows. The state space of the theory is a convex polytope, and reversible transformations must map it to itself. They therefore correspond to symmetries of the polytope. The polytope is in some way stellated, with the vertices corresponding to maximally non-local states having a different character from local ones. They are hence not connected by symmetries of the polytope. A two-dimensional caricature is shown in Figure 1.

The presentation proceeds as follows. We begin by formally introducing boxworld, then proceed to give the mathematical framework we will work with. This is essentially the standard generalized probabilistic framework, as used in [12, 15-17]. For clarity of exposition, in the main text we restrict to the case of two binary measurements $(M=2$, $K=2$ ) and give proofs of the main theorems for this case. The general case is deferred to the appendix, where the proofs are slightly more complicated but analogous.

Boxworld.-Recall that we have a system comprising $N$ subsystems and, on each subsystem, one of $M$ possible measurements can be applied (corresponding to different measurement devices), yielding one of $K$ possible outcomes (in the most general case, $K$ depends on the measurement). The local measurements are denoted $\left\{X_{0}, X_{1}, \ldots, X_{M-1}\right\}$. A measurement on the entire system made up of local measurements can then be described by a string $A_{1} \ldots A_{N}$, where $A_{i} \in\left\{X_{0}, X_{1}, \ldots, X_{M-1}\right\}$ specifies the measurement applied to the $i$ th subsystem. Similarly, the corresponding outcomes are denoted $a_{1} \ldots a_{N}$, with $a_{i} \in\{0,1, \ldots, K-1\}$. Measurement-outcome pairs are called effects, e.g. a measurement of $X_{1}$ giving outcome 3. A state is then a function $P:\left(a_{1} \ldots a_{N} \mid A_{1} \ldots A_{N}\right) \mapsto[0,1]$, which gives the probability of the effect that $A_{1} \ldots A_{N}$ is measured and gives outcomes $a_{1} \ldots a_{N}$. More general measurements are possible: a measurement is a collection of effects for which the sum of the outcome probabilities over the collection is 1 when acting on any state. Such measurements include procedures whereby the measurement performed on a particular subsystem depends on the outcomes of previous measurements, con- vex combinations of such procedures and more [14]. However, the statistics of the local measurements $A_{1} \ldots A_{N}$ are sufficient to uniquely determine the outcome probabilities of all measurements, and hence can be used to specify the state. This non-trivial assumption is known as the local observability principle [18].

Furthermore, the subsystems can be spatially separated, and hence we require that $P$ satisfies the non-signalling conditions, i.e. that

$$
\sum_{a_{i}=0}^{K-1} P\left(a_{1}, \ldots, a_{i}, \ldots, a_{N} \mid A_{1}, \ldots, A_{i}, \ldots, A_{N}\right)
$$

is independent of $A_{i}$. This implies that the marginal distribution on some set of subsystems is independent of the choice of measurement(s) on other subsystems.

Boxworld is a physical theory whose state space consists of any $P$ subject to: (i) $P$ takes values in $[0,1]$; (ii) $P$ is normalized in the obvious sense; and (iii) $P$ satisfies the nonsignalling conditions (1). The constraints $(i)$ - (iii) are such that the state space is a convex polytope, which turns out to have a non-trivial structure.

We first deal with the special case $M=K=2$ (the case of so-called gbits [12]). The corresponding state spaces (defined below) contain interesting non-local states, for example, non-local boxes with maximal Bell violating correlations. We label the measurements $X_{0}=X$ and $X_{1}=Z$.

Mathematical Framework.-We work in the generalized probabilistic framework (see e.g. [12, 15-17]). Here, states are represented as vectors embedded in a real vector space. Effects will also be represented as vectors, such that the probabilities of outcomes will be given by inner products between the relevant vectors. We begin with the case of a single system $(N=1)$. We choose three linearly independent vectors $X, Z, \underline{1} \in \mathbb{R}^{3}$. The vector $X$ is identified with $(1 \mid X)$, which is the effect that the $X$ measurement gives outcome 1 . We define a vector $\neg X:=\underline{1}-X$ and associate it with $(0 \mid X)$. The prefix $\neg$ may be interpreted as a negation. Lastly, the $\neg Z$ effect is defined analogously as $\neg Z:=\underline{1}-Z$. Because $X, Z, \underline{1}$ are linearly independent, for every state $P$, there is a unique vector $s \in \mathbb{R}^{3}$ representing $P$ in the sense that

$$
\langle X, s\rangle=P(1 \mid X), \quad\langle Z, s\rangle=P(1 \mid Z), \quad\langle\underline{1}, s\rangle=1 .
$$

It follows that $\langle\neg X, s\rangle=P(0 \mid X)$ and likewise for $Z$. We will refer to the set $\mathcal{P}^{(1)}=\{X, \neg X, Z, \neg Z\}$ as the singlesite extremal effects, for reasons that will become clear below. (Note that the quantum analogue of our effect vectors are projectors, and the inner product is analogous to the HilbertSchmidt scalar product, mapping states, $\rho$, and projectors, $\Pi$, to probabilities, $\operatorname{Tr}(\rho \Pi)$.)

The $N$-subsystem extremal effects $\mathcal{P}^{(N)}$ are defined to be the tensor products $A_{1} \otimes \cdots \otimes A_{N}$, where $A_{i} \in \mathcal{P}^{(1)}$ (the reason for this definition is that it recovers the full set of nonsignalling distributions for the state space, as will be shown in Lemma 1). We further define the identity on $N$ sites, $\underline{1}^{(N)}:=$ 
$\underline{1} \otimes \ldots \otimes 1$. A central object is the convex cone $\mathcal{K}^{(N)}$ generated by $\mathcal{P}^{(N)}$ [19]. This cone is the collection of all vectors which can be written as a linear combination of elements of $\mathcal{P}^{(N)}$ with non-negative coefficients. For any convex cone $\mathcal{K}$, one can associate a dual cone $\mathcal{K}^{*}=\{s \mid\langle A, s\rangle \geq 0 \forall A \in \mathcal{K}\}$. We will identify this with the set of unnormalized states.

Our interest in cones and duality stems from the following lemma, which characterizes the state space of boxworld in terms of the cone $\mathcal{K}^{(N)}$. It also implies the well-known result that there are no entangled effects in boxworld.

Lemma 1.-Let $\mathcal{S}^{(N)}$ be the set of vectors $s$ in the dual cone $\left(\mathcal{K}^{(N)}\right)^{*}$ which satisfy $\left\langle\underline{1}^{(N)}, s\right\rangle=1$. The space of (normalized) states in boxworld can be represented by $\mathcal{S}^{(N)}$.

Proof. We use the notation $\neg^{0} A:=A$ and $\neg^{1} A:=\neg A$ for $A \in\{X, Z\}$. The vectors $s \in \mathcal{S}^{(N)}$ will henceforth be called states; they satisfy $\left\langle\underline{1}^{(N)}, s\right\rangle=1$ and $\langle B, s\rangle \geq 0$ for all $B \in$ $\mathcal{K}^{(N)}$. To every state $s$, we associate a probability distribution $P$ via

$P\left(a_{1}, \ldots, a_{N} \mid A_{1}, \ldots, A_{N}\right):=\left\langle\neg^{a_{1}} A_{1} \otimes \ldots \otimes \neg^{a_{N}} A_{N}, s\right\rangle$

for $A_{i} \in\{X, Z\}$ and $a_{i} \in\{0,1\}$. First we show that every such $P$ is a valid non-signalling probability distribution. By definition, $P$ is non-negative. To see that it is normalized, note that we can decompose the identity

$$
\underline{1}^{(N)}=\sum_{x \in\{0,1\}^{N}} \neg^{x_{1}} A_{1} \otimes \neg^{x_{2}} A_{2} \otimes \ldots \otimes \neg^{x_{N}} A_{N}
$$

for any choices of $A_{i} \in\{X, Z\}$, so that

$$
\sum_{a_{1}, \ldots, a_{N}} P\left(a_{1}, \ldots, a_{N} \mid A_{1}, \ldots, A_{N}\right)=\left\langle\underline{1}^{(N)}, s\right\rangle=1 .
$$

To see that $P$ is non-signalling, consider

$$
\begin{aligned}
& \sum_{a_{i}} P\left(a_{1}, \ldots, a_{i}, \ldots, a_{N} \mid A_{1}, \ldots, A_{N}\right) \\
= & \left\langle\neg^{a_{1}} A_{1} \otimes \ldots \otimes A_{i} \otimes \ldots \otimes \neg^{a_{N}} A_{N}, s\right\rangle \\
& +\left\langle\neg^{a_{1}} A_{1} \otimes \ldots \otimes \neg A_{i} \otimes \ldots \otimes \neg^{a_{N}} A_{N}, s\right\rangle \\
= & \left\langle\neg^{a_{1}} A_{1} \otimes \ldots \otimes \underline{1} \otimes \ldots \otimes \neg^{a_{N}} A_{N}, s\right\rangle
\end{aligned}
$$

which is independent of $A_{i}$.

To show that every non-signalling distribution has an associated state, note that there is a unique vector $s \in\left(\mathbb{R}^{3}\right)^{\otimes N}$ such that $\left\langle\neg^{a_{1}} A_{1} \otimes \ldots \otimes \neg^{a_{N}} A_{N}, s\right\rangle=$ $P\left(a_{1}, \ldots, a_{N} \mid A_{1}, \ldots, A_{N}\right)$ for $\neg^{a_{i}} A_{i} \in\{X, Z, \neg X\}$ (since these effects span the space). It is then easy to see that the no-signalling property enforces consistency also in the case that $\neg^{a_{i}} A_{i}=\neg Z$ for some $i$. Non-negativity and normalization follow directly from the corresponding statements for the probability distribution $P$.

Transformations.-We now consider transformations in boxworld. First note that all allowed dynamical transformations in general probabilistic theories (reversible or not) are linear-this follows from the fact that they have to respect convex combinations, which correspond to probabilistic mixtures. For a general proof of this fact see [12].

The allowed transformations, $T$, are defined to be linear maps with the property that for all $s \in \mathcal{S}^{(N)}, T s \in \mathcal{S}^{(N)}$. A transformation is reversible if both $T$ and $T^{-1}$ are allowed transformations. It follows that a reversible transformation maps the state space $\mathcal{S}^{(N)}$ bijectively onto itself. Furthermore, since $T$ is a linear map, it is also the case that $T$ maps extremal states to extremal states. (More generally, one would only consider a transformation allowed if $T \otimes \mathbb{1}$ is also allowed-a condition analogous to complete positivity in quantum theory. However, our result applies without this additional requirement.)

Note that the states $s \in \mathcal{S}^{(N)}$ themselves do not have a physical meaning - only their scalar products with effects do, i.e. $\langle A, s\rangle$ (which are probabilities). Since $\langle A, T s\rangle=$ $\left\langle T^{\dagger} A, s\right\rangle$, the dynamics may equivalently be specified by means of the adjoint map $T^{\dagger}$. (In quantum theory, the analogue is passing from the Schrödinger to the Heisenberg picture.) Reversible transformations, $T$, map the state space bijectively onto itself and, likewise, the adjoint transformations $T^{\dagger}$ act accordingly on the cone of effects $\mathcal{K}^{(N)}$.

Lemma 2.-Adjoint reversible transformations $T^{\dagger}$ map the cone of effects $\mathcal{K}^{(N)}$ bijectively onto itself. Moreover, they map the set of extremal effects, $\mathcal{P}^{(N)}$, onto itself.

Proof. Any vector $t \in \mathcal{K}^{(N) *}$ can be written $t=\lambda s$ for some $\lambda \geq 0$ and some $s \in \mathcal{S}^{(N)}$. Then, for any outcome $B \in \mathcal{K}^{(N)}$, we have

$$
\left\langle T^{\dagger} B, t\right\rangle=\langle B, T t\rangle=\lambda\langle B, T s\rangle \geq 0,
$$

since $T s \in \mathcal{S}^{(N)}$. From the definition of the dual cone, it follows that $T^{\dagger} B \in\left(\mathcal{K}^{(N)}\right)^{* *}=\mathcal{K}^{(N)}$ (note that $\mathcal{K}^{* *}=\mathcal{K}$ for every closed convex cone $\mathcal{K}$ (cf. [19])). Therefore, $T^{\dagger}$ maps the cone of effects $\mathcal{K}^{(N)}$ into itself. The same argument applies to the inverse $\left(T^{\dagger}\right)^{-1}=\left(T^{-1}\right)^{\dagger}$, hence the cone is mapped bijectively onto itself.

Since it is a convex cone, $\mathcal{K}^{(N)}$ is completely characterized by its extremal rays. By linearity, $T^{\dagger}$ maps the extremal rays of $\mathcal{K}^{(N)}$ onto themselves. From the definition of $\mathcal{K}^{(N)}$, we know that the cone is the convex hull of the $4^{N}$ rays formed by all $A \in \mathcal{P}^{(N)}$. It is elementary to check that these are indeed the extremal rays. Therefore, for every $A \in \mathcal{P}^{(N)}$, there exists an $A^{\prime} \in \mathcal{P}^{(N)}$ and a non-negative number $\lambda$ such that $T^{\dagger}(A)=\lambda A^{\prime}$. To see that $\lambda$ must equal 1 , observe that for every $B \in \mathcal{P}^{(N)}$, there exist (product) states $s_{0}, s_{1} \in \mathcal{S}^{(N)}$ such that $\left\langle s_{0}, B\right\rangle=0$ and $\left\langle s_{1}, B\right\rangle=1$. Since this holds in particular for both $A$ and $A^{\prime}$, it follows that $\lambda=1$.

Orthogonal representation of transformations. - There are $4^{N}$ extremal effects, and thus $4^{N}$ ! permutations acting on $\mathcal{P}^{(N)}$. We go on to show that only a tiny fraction of those is actually realizable in boxworld. It will be convenient to use a specific representation of $X, Z$ and $\underline{1}$ :

$$
X=(1 / 2,1 / \sqrt{2}, 0), \quad Z=(1 / 2,0,1 / \sqrt{2}), \quad \underline{1}=(1,0,0) .
$$

Lemma 3.-With respect to the representation above, it holds that any reversible transformation $T$ is orthogonal, i.e. 
on $N$ subsystems, $T^{\dagger} T=\mathbb{1}_{3^{N}}$, where $\mathbb{1}_{d}$ is the $d$-dimensional identity matrix.

Proof. First observe that with this choice, $\sum_{A \in \mathcal{P}^{(1)}}|A\rangle\langle A|=$ $\mathbb{1}_{3}$ and hence (since $\mathcal{P}^{(N)}$ factorizes) $\sum_{A \in \mathcal{P}^{(N)}}|A\rangle\langle A|=$ $\mathbb{1}_{3^{N}}$. Then, since $T^{\dagger}$ permutes the extremal effects, $T^{\dagger} T=$ $T^{\dagger}\left(\sum_{A \in \mathcal{P}^{(N)}}|A\rangle\langle A|\right) T=\sum_{A \in \mathcal{P}^{(N)}}|A\rangle\langle A|=\mathbb{1}_{3^{N}}$.

The fact that $T$ (and thus $T^{\dagger}$ ) is orthogonal, gives rise to a host of invariants. If one picks any two extremal effects $Q, R \in \mathcal{P}^{(N)}$, then clearly their inner product is a conserved quantity: $\langle Q, R\rangle=\left\langle T^{\dagger} Q, T^{\dagger} R\right\rangle$. However, $|\langle Q, R\rangle|=$ $4^{-N} 3^{N-d_{H}(Q, R)}$, where $d_{H}(Q, R)$ is the Hamming distance between $Q$ and $R$, i.e. the number of places at which $Q$ and $R$ differ. Thus the Hamming distance of extremal effects is a conserved quantity: $d_{H}(Q, R)=d_{H}\left(T^{\dagger} Q, T^{\dagger} R\right)$.

It is well-known in the theory of error correction [20] that the set of maps on finite strings which preserve the Hamming distance is highly restricted: the group of those maps is generated by local transformations and permutations of sites only (for a proof, see the Appendix). Thus $T^{\dagger}$ acts as such an operation on $\mathcal{P}^{(N)}$. Moreover, since the states in $\mathcal{P}^{(N)}$ span the entire space, the action on this set is sufficient to completely specify $T^{\dagger}$.

Furthermore, it is straightforward to show that the set of allowed local operations comprises exchanging $X$ and $Z$ (relabelling measurements), exchanging $X$ and $\neg X$ (relabelling the outcome upon input $X$ ), exchanging $Z$ and $\neg Z$ (relabelling the outcome upon input $Z$ ) and combinations thereof (see Lemma 8 in the Appendix, for a proof in the general case).

Main results.-Combining all the previous results proves the following theorem in the special case of $M=2$ measurements with $K=2$ outcomes (a full proof for all $M \geq 2$ and $K \geq 2$ is given in the appendix):

Theorem 1.-Every reversible transformation on a system comprising $N$ subsystems in boxworld, with $M \geq 2$ measurements at every subsystem each having $K \geq 2$ outcomes, is a permutation of subsystems, followed by local relabellings of measurements and their outcomes.

Furthermore, we show the following:

Theorem 2.- In boxworld, every reversible transformation maps pure product states to pure product states. This is true even if the system is coupled to an arbitrary number of classical systems, and if the number of devices and outcomes varies from subsystem to subsystem.

Before giving the proof, we need to slightly extend the notion of outcome vectors to the general case. We denote the set of extremal effects for the $i$ th subsystem by $\mathcal{P}^{i}=\left\{X_{m}^{i}(k)\right\}$, where $m$ labels the measurements (the number of different $m$ s may depend on $i$ ) and $k$ the corresponding outcomes (the number of different $k \mathrm{~s}$ may depend on $m$ and on $i$ ). These vectors satisfy $\sum_{k} X_{m}^{i}(k)=\underline{1}^{i}$, where $\underline{1}^{i}$ represents the identity. Except for these relations, no linear dependencies occur.
The identity on the full system is then $\underline{1}^{(N)}:=\underline{1}^{1} \otimes \ldots \otimes \underline{1}^{N}$, and the extremal effects are $\mathcal{P}^{(N)}:=\overline{\mathcal{P}}^{1} \otimes \ldots \otimes \mathcal{P}^{N}$. The convex cone $\mathcal{K}^{(N)}$ and the state space $\mathcal{S}^{(N)}$ are defined analogously to the binary case previously described. The statements and proofs of Lemmas 1 and 2 remain valid in this more general case, hence, in particular, adjoint reversible transformations map $\mathcal{P}^{(N)}$ onto itself.

Proof. To complete the proof of Theorem 2, note that a state $s \in \mathcal{S}^{(N)}$ is a pure product state (that is, of the form $s=$ $s_{1} \otimes \ldots \otimes s_{N}$, where all $s_{i}$ are pure) if and only if $\langle A, s\rangle \in$ $\{0,1\}$ for all extremal effects $A \in \mathcal{P}^{(N)}$ (a proof is given in Lemma 9]. Suppose that $s$ is a pure product state and $T$ a reversible transformation, then

$$
\langle A, T s\rangle=\left\langle T^{\dagger} A, s\right\rangle \in\{0,1\} \text { for all } A \in \mathcal{P}^{(N)},
$$

which proves that Ts must also be a pure product state.

Note that Theorem 1 does not, in general, apply to the case of site-dependent numbers of measurements. For example, suppose that we have two sites, where the first has two binary measurements, $X$ and $Z$, and the second allows only a single binary measurement, $Y$. (In other words, a gbit is coupled to a classical bit.) It is then straightforward to construct a reversible CNOT operation, where the classical bit is the control bit. For example, there is an adjoint reversible transformation that acts as

$$
A \otimes Y \mapsto A \otimes Y, \quad A \otimes \neg Y \mapsto \neg A \otimes \neg Y
$$

for all $A \in\{X, Z, \neg X, \neg Z\}$.

In the case of a system composed of several classical subsystems, Theorem 1 also does not hold — the dynamics in such a case is non-trivial. Nevertheless, Theorem 2 does apply to this case-it remains impossible to prepare entangled states from separable ones.

Conclusions. - We have shown that the set of reversible operations in boxworld is trivial: the only possible operations relabel subsystems, local measurements and their outcomes. In particular, there is no boxworld analogue of an entangling unitary in quantum theory, one cannot reversibly prepare nonlocal states from separable ones, nor perform useful computations reversibly.

In addition, the results have consequences for the interplay between dynamics and measurements in boxworld: suppose we have a system comprising a particle, $A$, and two observers, $B$ and $C$, initially in an uncorrelated tripartite product state. In quantum theory, if $B$ measures $A$, but $C$ does not take part in the interaction, then $C$ can model the corresponding dynamics by a unitary transformation on the $A B$-system. That is, $C$ can view the whole interaction as reversible while retaining the ability to correctly predict the outcome probabilities of any future measurements. (Theories with such a property might be called fundamentally reversible.) In boxworld, on the other hand, this is not true: $B$ 's measurement on $A$ would have to create correlations between $A$ and $B$, but this could never be achieved by a reversible transformation. Hence $C$ would have 
to model the $A B$-measurement using irreversible dynamics, even if $C$ did not take part in the interaction itself.

It would be interesting to extend our result to explore which state spaces are compatible with fundamentally reversible theories in this sense, or with theories that are transitive, i.e. that every pure state can be reversibly mapped to any other. This property has been used by Hardy as an axiom for quantum theory [16]. Both conditions seem to strongly restrict the possible geometry of the state space, and an interesting open question is how non-local such theories can be.
Acknowledgments. - We are grateful to Howard Barnum, Volkher Scholz, and Reinhard Werner for interesting discussions and to Nicholas Harrigan for comments which improved the presentation. OD would like to thank Matthew Leifer for introducing him to generalized probabilistic theories, and MM would like to thank Christopher Witte for the same reason and for the quadratic cocktail model of a gbit. RC and OD acknowledge support from the Swiss National Science Foundation (grant No. 200021-119868). DG's research is supported by the EU (CORNER).
[1] J. Bell, Cambridge University Press, Cambridge (1987).

[2] A. Aspect, Nature 398, 189 (1999).

[3] A. Ekert, Phys. Rev. Lett., 67, 661, (1991).

[4] V. Scarani et. al., arXiv:0802.4155 (2008).

[5] L. A. Khalfin and B. S. Tsirelson, World Scientific, Singapore, 441 (1985).

[6] B. S. Tsirelson, Hadronic Journal Supp. 8:4, 329 (1993).

[7] S. Popescu, D. Rohrlich, Found. Phys. 24379 (1994).

[8] W. van Dam, quant-ph/0501159 (2005).

[9] G. Brassard, H. Buhrman, N. Linden, A. Méthot, A. Tapp and F. Unger, Phys. Rev. Lett., 96, 250401, (2006).

[10] H. Buhrman et al., Proc. Royal Society A 4621919 (2006).

[11] M. Pawlowski et al., arXiv:0905.2292 (2009).

[12] J. Barrett, Phys. Rev. A, 75, 032304 (2007).

[13] T. Short, S. Popescu, and N. Gisin, Phys. Rev. A 73, 012101 (2006)

[14] T. Short and J. Barrett, arXiv:0909.2601 (2009).

[15] H. Barnum, J. Barrett, M. Leifer, and A. Wilce, quant-ph/0611295 (2006).

[16] L. Hardy, quant-ph/0101012 (2001).

[17] A. S. Holevo, "Probabilistic and Statistical Aspects of Quantum Theory", North Holland (1982).

[18] G. M. D’Ariano, quant-ph/0701217 (2007).

[19] C. D. Aliprantis, R. Tourky, "Cones and Duality", American Mathematical Society (2007).

[20] P. Kaski, P. R. J. Östergård, "Classification Algorithms for Codes and Designs", Springer (2006).

\section{Appendix}

This appendix contains the proof of Theorem 1: Every reversible transformation on a system comprising $N$ subsystems in boxworld, with $M \geq 2$ measurements at every subsystem each having $K \geq 2$ outcomes, is a permutation of subsystems, followed by local operations.

The proof idea is the same as in the case $M=K=2$ : find a particular representation of the vectors $X_{m}(k)$ and $\underline{1}$ (corresponding to the previous vectors $X, Z, \neg X$ and $\neg Z$ and $\underline{1}$ ) such that reversible transformations are orthogonal, and such that the scalar products of those vectors yield useful invariants. We recall that the dual of reversible transformations preserve the cone of effects and so permute extremal effects (this is Lemma 2 applied to this case).

We start with the following observation:

Lemma 4. For every $N \in \mathbb{N}$, there exist unit vectors

$$
\begin{aligned}
\left\{w_{i}\right\}_{i=1}^{N+1} \text { in } \mathbb{R}^{N} \text { with the properties } & \text { - }\left\langle w_{i}, w_{j}\right\rangle=-\frac{1}{N} \text { if } i \neq j, \\
& -\sum_{i=1}^{N+1} w_{i}=0 \text {, and } \\
& -\frac{1}{N+1} \sum_{i=1}^{N+1}\left|w_{i}\right\rangle\left\langle w_{i}\right|=\frac{1}{N} \mathbb{1}_{N} .
\end{aligned}
$$

Proof. Rather than giving the vectors explicitly, we construct them implicitly from the standard $N$-simplex in $\mathbb{R}^{N+1}$ : let $e_{i}$ be the $i$ th standard unit vector in $\mathbb{R}^{N+1}$, and $c$ the center of those vectors, that is $c:=\frac{1}{N+1} \sum_{i=1}^{N+1} e_{i}$. Define $v_{i}:=e_{i}-c$, so that the angles between those vectors $(i \neq j)$ are $\frac{\left\langle v_{i}, v_{j}\right\rangle}{\left\|v_{i}\right\|\left\|v_{j}\right\|}=-\frac{1}{N}$. By construction, we have $\sum_{i=1}^{N+1} v_{i}=0$, so the vectors are linearly dependent. The set $\left\{w_{i}\right\}_{i=1}^{N+1}$ are then the vectors resulting from embedding normalized versions of the $v_{i}$ s isometrically into $\mathbb{R}^{N}$. The first two claimed equalities follow immediately. The third can be confirmed by computing ${ }^{1}$

$$
\| \frac{1}{N+1} \sum_{i=1}^{N+1}\left|w_{i}\right\rangle\left\langle w_{i}\right|-\frac{1}{N} \mathbb{1}_{N} \|_{2}^{2}=0,
$$

which involves only scalar products of the form $\left\langle w_{i}, w_{j}\right\rangle$.

The vectors $X_{m}(k)$ representing the $k$ th measurement outcomes for the $m$ th measurement (counting from zero) can be constructed as follows:

- Choose $\underline{1} \neq 0$ arbitrarily,

- choose $X_{m}(0), X_{m}(1), \ldots, X_{m}(K-2)$ for all $m$ such that all obtained vectors are linearly independent,

- define $X_{m}(K-1)$ as $\underline{1}-\sum_{k=0}^{K-2} X_{m}(k)$.

We choose these in a particular way in order to simplify the subsequent argument: the single-site effects will be vectors in $\mathbb{R}^{M(K-1)+1}=\left(\mathbb{R}^{M} \otimes \mathbb{R}^{K-1}\right) \oplus \mathbb{R}$. Let $e_{m}$ denote the $m$ th

\footnotetext{
${ }^{1}$ The Schatten 2-norm is defined by $\|A\|_{2}^{2}:=\operatorname{tr}\left(A A^{\dagger}\right)$.
} 
standard unit vector in $\mathbb{R}^{M}$, and $\underline{1}$ be the unit vector on the direct sum space, $\mathbb{R}$. Then, define

$$
X_{m}(k):=\sqrt{\frac{M(K-1)}{K^{2}}} e_{m+1} \otimes w_{k+1}+\frac{1}{K} \underline{1}
$$

for $0 \leq m \leq M-1$ and $0 \leq k \leq K-1$. Useful properties of these vectors are given in the following lemma.

Lemma 5. In the representation given above, we have

$$
\begin{gathered}
\sum_{k=0}^{K-1} X_{m}(k)=\underline{1} \\
\sum_{m=0}^{M-1} \sum_{k=0}^{K-1}\left|X_{m}(k)\right\rangle\left\langle X_{m}(k)\right|=\frac{M}{K} \mathbb{1}, \text { and } \\
\left\langle X_{m}(k), X_{m^{\prime}}\left(k^{\prime}\right)\right\rangle=\frac{1}{K^{2}}\left\{\begin{array}{cl}
1 & m \neq m^{\prime} \\
1-M & m=m^{\prime}, k \neq k^{\prime} \\
1+M(K-1) & m=m^{\prime}, k=k^{\prime}
\end{array}\right.
\end{gathered}
$$

Moreover, reversible transformations are orthogonal with respect to this representation.

Proof. The three equations can be verified by direct calculation. That reversible transformations are orthogonal is simply the extension of Lemma 3 to the present case.

We remark that the inner product $1-M$ for $m=m^{\prime}, k \neq$ $k^{\prime}$ is the reason why Theorem 2 does not hold in the case of classical systems $(M=1)$. In the following, we will assume that $M \geq 2$.

We now consider an adjoint reversible transformation, $T^{\dagger}$. Note that for all $s \in \mathcal{S}^{(N)}$,

$$
1=\left\langle\underline{1}^{(N)}, T s\right\rangle=\left\langle T^{\dagger} \underline{1}^{(N)}, s\right\rangle,
$$

from which it follows that $T^{\dagger} \underline{1}^{(N)}=\underline{1}^{(N)}$. Moreover, we have the following property:

Lemma 6. Let $Q, R \in \mathcal{P}^{(N)}$ be two extremal effects that differ at exactly one site, and let $T^{\dagger}$ be an adjoint reversible transformation. Then, $T^{\dagger} Q$ and $T^{\dagger} R$ also differ at exactly one site.

Proof. Since $Q$ and $R$ factorize, we can compute the inner product $\langle Q, R\rangle$ termwise. Let $i$ be the site where $Q$ and $R$ differ, and let $Q_{i}$ and $R_{i}$ be the corresponding factors.

First, consider the case that $Q_{i}$ and $R_{i}$ represent different outcomes of the same measurement. Then, the inner product is the negative value

$$
K^{2 N}\langle Q, R\rangle=(1-M)(1+M(K-1))^{N-1}
$$

which is the smallest value that can possibly be attained. Hence $K^{2 N}\left\langle T^{\dagger} Q, T^{\dagger} R\right\rangle$ has the same value, such that $T^{\dagger} Q$ and $T^{\dagger} R$ also differ at a single site only (where they refer to different outcomes of the same measurement).

The alternative case is where $Q_{i}$ and $R_{i}$ represent outcomes of different measurements. Note that $\underline{1}-Q_{i}-R_{i} \notin \mathcal{K}$ and hence $\underline{1}^{(N)}-Q-R \notin \mathcal{K}^{(N)}$. Since $T^{\dagger}$ preserves $\underline{1}^{(N)}$ and maps the cone bijectively to itself (cf. Lemma 2), we have $\underline{1}^{(N)}-T^{\dagger} Q-T^{\dagger} R \notin \mathcal{K}^{(N)}$, from which it follows that $T^{\dagger} Q$ and $T^{\dagger} R$ correspond to outcomes of different measurements on at least one factor. Furthermore,

$$
K^{2 N}\langle Q, R\rangle=(1+M(K-1))^{N-1}
$$

is preserved. This is the largest value that can be attained subject to the constraint that they represent outcomes of different measurements on at least one factor. It follows that $T^{\dagger} Q$ and $T^{\dagger} R$ are identical in all but one tensor factor.

The proof of Theorem 1 is now completed using some properties of the Hamming distance. The list of local effects (listing the measurement-outcome pairs at the successive sites) can be used to form a string in $\mathbb{Z}_{d}^{N}$, where $d=M K$. The Hamming distance between two strings, $Q$ and $R$, is defined by

$$
d_{H}(Q, R):=\left|\left\{i: Q_{i} \neq R_{i}\right\}\right| .
$$

Lemma 6 shows that if $Q, R \in \mathcal{P}^{(N)}$ are two arbitrary extremal effects with $d_{H}(Q, R)=1$, then the transformed effects satisfy $d_{H}\left(T^{\dagger} Q, T^{\dagger} R\right)=1$.

In fact, all reversible operations that preserve Hamming distance 1 preserve the Hamming distance between all effects. Furthermore, the set of Hamming distance preserving transformations can be expressed as combinations of permutations of subsystems and local permutations (see for example Theorem 3.54 of [20]). We give a proof of this for completeness.

Lemma 7. Let $\mathcal{A}$ be a finite alphabet, with $\mathcal{A}^{N}$ the set of length- $N$ words. Further, let $G_{\Pi} \cong S_{N}$ be the set of permutations of letters and $G_{L} \cong S_{|A|}^{N}$ be the group of local transformations of $\mathcal{A}^{N}$, which act independently at each position.

Assume that $T^{\dagger}: \mathcal{A}^{N} \rightarrow \mathcal{A}^{N}$ is invertible. If $T^{\dagger}$ has the property that for all $s, t \in \mathcal{A}^{N}, d_{H}(s, t)=1 \Longrightarrow$ $d_{H}\left(T^{\dagger} s, T^{\dagger} t\right)=1$, then $T^{\dagger}$ is a composition of operations from $G_{\Pi}$ and $G_{L}$.

Proof. Choose an arbitrary set $a_{i} \in A$, for $i=1, \ldots, N$. Set $s=\left(a_{1}, \ldots, a_{N}\right)$. Left-multiplying $T^{\dagger}$ by a local operation if necessary, we may assume that $T^{\dagger}(s)=s$.

For $i \in 1 \ldots N$, consider the set, $L_{i}$ of words of the form

$$
L_{i}=\left(a_{1}, \ldots, a_{i-1}, A_{i}, a_{i+1}, \ldots, a_{N}\right),
$$

i.e. $s$ and strings that differ from $s$ only at position $i$. Because the elements of $L_{i}$ all have mutual Hamming distance equal to one, there must be a function $\pi$ such that $T^{\dagger}\left(L_{i}\right)=L_{\pi(i)}$. Since $T^{\dagger}$ is invertible, $\pi$ is a permutation, which may be thought of as an element of $G_{\Pi}$. Because $\left(\pi^{-1}\right) T^{\dagger}\left(L_{i}\right)=L_{i}$ for all $i$, there is no loss of generality in assuming that $T^{\dagger}$ takes $L_{i}$ to itself. Employing yet another local operation if necessary, we may even assume that $T$ acts like identity on all elements of $L_{i}$, and hence on all strings with Hamming distance 1 to $s$.

Define the weight of an element $t \in \mathcal{A}^{N}$ to be wt $(t)=$ $d_{H}(t, s)$. What we have shown so far amounts to the fact that 
$T^{\dagger}$ fixes all words of weight zero and one. Next, we prove by induction that $T^{\dagger}$ fixes the words of any weight $w$ (and hence all of $\left.\mathcal{A}^{N}\right)$.

Assume the claim has been established for weights up to $w-1$. If $t$ has weight $w>1$, it is uniquely specified by the $w$ words $r_{i}$ which have weight wt $\left(r_{i}\right)=w-1$ and Hamming distance $d_{H}\left(r_{i}, t\right)=1$ to $t$ (in fact, any two words $r_{i}, r_{j}$ from this set are sufficient to specify $t$ ). But since the weights of all of the $r_{i}$, and $d_{H}\left(r_{i}, t\right)$ are preserved by $T^{\dagger}$ by the induction hypothesis, $T^{\dagger}$ must fix $t$.

It follows that all transformations in boxworld can be formed by composing subsystem permutations and local permutations. However, the set of allowed local permutations is further restricted (Hamming distance preservation is a necessary but not sufficient condition on $T^{\dagger}$ ):

Lemma 8. The only local reversible operations allowed in boxworld are relabellings of measurements and their outcomes (separately for each measurement). Furthermore, all possible local relabellings are allowed transformations, regardless of the total number of subsystems, $N$.

Proof. Recall (4) and note that these are the only combinations of extremal effects that sum to $\underline{1}$ : otherwise the identity 1 could be decomposed into a sum involving two effects $X_{m}(k)$ and $X_{m^{\prime}}\left(k^{\prime}\right)$ with $m \neq m^{\prime}$, so there would be a state $s$ with $\left\langle X_{m}(k), s\right\rangle=\left\langle X_{m^{\prime}}\left(k^{\prime}\right), s\right\rangle=1$, for which $\langle\underline{1}, s\rangle \geq 2$, a contradiction.

Consider a measurement $m$, then

$$
\underline{1}=T^{\dagger} \underline{1}=T^{\dagger} \sum_{k} X_{m}(k)=\sum_{k} T^{\dagger} X_{m}(k),
$$

so each member of $\left\{T^{\dagger} X_{m}(k): 0 \leq k \leq K(m)-1\right\}$ must correspond to the same measurement. Hence all local reversible adjoint transformations permute the measurements, and, for each measurement separately, permute the outcomes.

To see that all permutations are allowed if the number of outcomes $K$ is the same for every measurement $m$, note that the representation of $X_{m}(k)$ as in (3) on $\left(\mathbb{R}^{M} \otimes \mathbb{R}^{K-1}\right) \oplus \mathbb{R}$ permits that all those permutations are implemented as allowed linear (hence orthogonal) transformations: relabelling the measurements corresponds to permuting the standard unit vectors $e_{m}$ of $\mathbb{R}^{M}$ (constituting an $M$-dimensional irreducible representation of the symmetric group $S_{M}$ ), while relabelling the outcomes corresponds to symmetry transformations of the $(K-1)$-simplex in $\mathbb{R}^{K-1}$ with $K$ vertices $w_{k}(\mathrm{a}(K-1)$ dimensional irreducible representation of $S_{K}$ ).

In the case that the number of outcomes $K=K(m)$ depends on the measurement $m$, the vector space carrying the local effects will analogously be $\bigoplus_{m=0}^{M-1} \mathbb{R}^{K(m)-1} \oplus \mathbb{R}$. This allows us to represent the permutation of outcomes linearly, as before, while the permutations of measurements $m$ and $m^{\prime}$ with $K(m)=K\left(m^{\prime}\right)$ correspond to permutations of direct summands.

We have thus proven that every local relabelling transformation $T^{\dagger}$ is an allowed transformation in boxworld. It remains to show that $T^{\dagger}$ is allowed if the single system is coupled to others (i.e. that $T^{\dagger} \otimes \mathbb{1}$ is an allowed transformation). (The analogue in quantum theory is to prove complete positivity). This follows from the fact that local relabellings preserve the no-signalling, positivity and normalization constraints, such that $T \otimes \mathbb{1}$ maps the no-signalling polytope (that is, the state space) onto itself.

In the final part of the appendix, we prove that a state $s$ is a pure product state if and only if all the probabilities $\langle A, s\rangle$ are either 0 or 1 with respect to extremal effects, $A$ (in the most general case that the number of measurements and outcomes varies from site to site). This has been used in the proof of Theorem 2.

Lemma 9. Let $s \in S^{(N)}$ be a normalized state on $N$ arbitrary boxworld subsystems (some of which may be classical). Then, $s$ is a pure product state if and only if $\langle A, s\rangle \in\{0,1\}$ for all extremal effects $A=A_{1} \otimes \ldots \otimes A_{N} \in \mathcal{P}^{(N)}$.

Proof. If $s=s_{1} \otimes \ldots \otimes s_{N}$ is a product of pure states, then $\left\langle A_{i}, s_{i}\right\rangle \in\{0,1\}$ for every $i$, such that $\langle A, s\rangle$ is either 0 or 1 . It remains to prove the converse. Suppose that $s$ is any state with $\langle A, s\rangle \in\{0,1\}$ for all $A \in \mathcal{P}^{(N)}$. The idea is to construct a pure product state $\tilde{s}$ with $\langle A, s\rangle=\langle A, \tilde{s}\rangle$ for all $A$, which proves that $s=\tilde{s}$. To this end, note that the decomposition of the identity given in (2) has the following generalization: if the $m_{i}$ are arbitrary local measurement devices, then

$$
\underline{1}^{(N)}=\sum_{k_{1}, \ldots, k_{N}} X_{m_{1}}\left(k_{1}\right) \otimes X_{m_{2}}\left(k_{2}\right) \otimes \ldots \otimes X_{m_{N}}\left(k_{N}\right)
$$

where the sum is over all outcomes (the number of outcomes may depend on the measurement). It follows that

$$
1=\sum_{k_{1}, \ldots, k_{N}}\left\langle X_{m_{1}}\left(k_{1}\right) \otimes X_{m_{2}}\left(k_{2}\right) \otimes \ldots \otimes X_{m_{N}}\left(k_{N}\right), s\right\rangle,
$$

so exactly one of the addends must be 1 , while all others are 0 . Hence, to every string $\mathbf{m}=\left(m_{1}, m_{2}, \ldots, m_{N}\right)$ describing local choices of measurements, there is a unique string of corresponding outcomes $\mathbf{k}(\mathbf{m})=\left(k_{1}, k_{2}, \ldots, k_{N}\right)$ such that $\left\langle X_{m_{1}}\left(k_{1}\right) \otimes \ldots \otimes X_{m_{N}}\left(k_{N}\right), s\right\rangle=1$, while the inner product is 0 for all other outcome combinations. It remains to show that each $k_{i}$ only depends on $m_{i}$; in this case, we can construct a state $\tilde{s}=\tilde{s}_{1} \otimes \ldots \otimes \tilde{s}_{N}$, generating the same probabilities as $s$, factor by factor. So suppose there were strings $\mathbf{m}$ and $\tilde{\mathbf{m}}$ with $m_{i}=\tilde{m}_{i}$, but $k_{i} \neq \tilde{k}_{i}$, (where $\left.\tilde{\mathbf{k}}:=\mathbf{k}(\tilde{\mathbf{m}})\right)$ then

$$
\begin{aligned}
1= & \left\langle\underline{1} \otimes \ldots \otimes \underline{1} \otimes \sum_{k^{\prime}} X_{m_{i}}\left(k^{\prime}\right) \otimes \underline{1} \otimes \ldots \otimes \underline{1}, s\right\rangle \\
\geq & \left\langle\underline{1} \otimes \ldots \otimes \underline{1} \otimes X_{m_{i}}\left(k_{i}\right) \otimes \underline{1} \otimes \ldots \otimes \underline{1}, s\right\rangle \\
& +\left\langle\underline{1} \otimes \ldots \otimes \underline{1} \otimes X_{\tilde{m}_{i}}\left(\tilde{k}_{i}\right) \otimes \underline{1} \otimes \ldots \otimes \underline{1}, s\right\rangle \\
\geq & \left\langle X_{m_{1}}\left(k_{1}\right) \otimes \ldots \otimes X_{m_{N}}\left(k_{N}\right), s\right\rangle \\
& +\left\langle X_{\tilde{m}_{1}}\left(\tilde{k}_{1}\right) \otimes \ldots \otimes X_{\tilde{m}_{N}}\left(\tilde{k}_{N}\right), s\right\rangle \\
= & 2
\end{aligned}
$$

where the last inequality follows by applying the decomposition of the identity (6) to $N-1$ factors. This is a contradiction. 